\title{
Long Term Efficacy and Safety of Herring Roe Oil in the Treatment of Psoriasis, a 39-week Open-label Extension Study
}

\author{
Kaare Steinar Tveit ${ }^{1, ~}{ }^{*}$, Karl Albert Brokstad ${ }^{2}$, Rolf Kristian Berge ${ }^{2,3}$, Per Christian Saeboe ${ }^{4}$, \\ Hogne Hallaraaker ${ }^{4}$, Stian Brekke ${ }^{5}$, Nils Meland ${ }^{5}$, Bodil Bjoerndal ${ }^{2,6}$ \\ ${ }^{1}$ Department of Dermatology, Haukeland University Hospital, Bergen, Norway \\ ${ }^{2}$ Department of Clinical Science, University of Bergen, Bergen, Norway \\ ${ }^{3}$ Department of Heart Disease, Haukeland University Hospital, Bergen, Norway \\ ${ }^{4}$ Department of Product Development, Arctic Nutrition AS, Oersta, Norway. \\ ${ }^{5}$ Department of Regulatory Affairs and Biometrics, Smerud Medical Research International AS, Oslo, Norway \\ ${ }^{6}$ Department of Sport, Food and Natural Sciences, Western Norway University of Applied Sciences, Bergen, Norway
}

Email address:

kare.steinar.tveit@helse-bergen.no (K. S. Tveit)

${ }^{*}$ Corresponding author

\section{To cite this article:}

Kaare Steinar Tveit, Karl Albert Brokstad, Rolf Kristian Berge, Per Christian Saeboe, Hogne Hallaraaker, Stian Brekke, Nils Meland, Bodil Bjoerndal. Long Term Efficacy and Safety of Herring Roe Oil in the Treatment of Psoriasis, a 39-week Open-label Extension Study. International Journal of Clinical and Experimental Medical Sciences. Vol. 7, No. 1, 2021, pp. 13-20. doi: 10.11648/j.ijcems.20210701.13

Received: December 22, 2020; Accepted: January 6, 2021; Published: January 22, 2021

\begin{abstract}
The effect of omega-3 poly-unsaturated fatty acid supplements in patients with Psoriasis vulgaris has previously been investigated, but interventions varied in source, composition, dose, administration route and duration of treatment. The observed beneficial effects in patients with Psoriasis vulgaris using herring roe oil (HRO) as a dietary supplement prompted the conduct of this investigation. We monitored the longer-term efficacy and safety of as oral treatment for patients with plaque psoriasis originally included with a Psoriasis Area Severity Index (PASI) < 10 in a 39week open-label extension period following a 26 week double-blind randomised and placebo controlled study (ClinicalTrials.gov: NCT03359577, date: 02 December 2017). All patients in the randomised study who were still participating at week 26 were invited to continue on active treatment. Fifty-eight of 64 patients included in the randomised study, were all treated with HRO through the extension period; 28 subjects from the HRO group and 30 subjects from the placebo group. Change (mean \pm standard deviation; SD) in the PASI score from baseline in the 26 week double-blind period was $-2.13 \pm 2.57$ in the HRO-HRO group and $-0.63 \pm 1.87$ in the placebo-HRO group, and the difference between the groups was statistically significant $(\mathrm{p}<0.05)$. At conclusion of the entire 65 -week period (15 months), the change in PASI was $3.44 \pm 1.83$ in the HRO-HRO group and $-3.06 \pm 1.69$ in the placebo-HRO group. The secondary variables showed decreasing disease symptoms and clinically meaningful patient reported outcomes as shown with a mean reduction in Dermatological Life Quality Index (DLQI) score of $>4$ points. The most commonly reported adverse events in both groups were gastrointestinal in nature. No serious adverse reactions were reported.
\end{abstract}

Keywords: Herring Roe Oil, Psoriasis, Omega-3, Phospholipids

\section{Introduction}

Psoriasis is an immune-mediated inflammatory skin disease with complex pathogenesis and comorbidities including cardiovascular disease [1]. Has been used as a food supplement for several years and has previously been demonstrated to be bioavailable with beneficial effects on plasma lipids $[2,3]$. The oil extract contains phospholipid 
esters of omega-3 fatty acids including eicosapentaenoic acid (EPA) and docosahexaenoic acid (DHA) for which antiinflammatory effects have been previously demonstrated [4, $5]$.

We explored the efficacy of HRO in a randomised, double blind, placebo controlled clinical study in psoriasis patients with baseline PASI score $<10$ [6]. The randomised study period lasted for 26 weeks and all eligible patients were invited to continue a 39 -week, open-label, active treatment period. In the randomised study, a statistically significant reduction in the mean PASI score from baseline to week 26 was shown in the HRO treatment group compared to the placebo group. Although statistically significant improvements in patient reported outcome measures (PROMs) were not achieved compared to placebo, there was a decrease indicating self perceived disease improvement. In the HRO-HRO group $(\mathrm{n}=28)$ reductions from baseline to week 26 in mean PROMs ranged from 25 to $41 \%$, whereas the corresponding reductions in the placebo-HRO group $(n=30)$ ranged from 5 to $23 \%$.

The open-label, active extension period was conducted for exploratory evaluation of long term safety and efficacy. Here the clinical results of the open-label extension period are described.

\section{Materials and Methods}

\subsection{Subjects and Study Design}

Out of the 64 patients enrolled in a randomized, 26 weeks, single centre, placebo controlled, double blind study to explore the effects of, 58 continued and completed the 39week extension study. The study was approved by the Regional Committees for Medical and Health Research Ethics in western Norway (REK Vest, 2017/938) and it was performed at the Department of Dermatology, Haukeland University Hospital, Bergen, Norway.

Patients with stable psoriasis for 6 months and with PASI scores less than 10 were eligible to be included in the randomised study. Patients on stable local anti-psoriatic maintenance treatment for more than two months before study start continued this treatment. There were no limitations on the use of unmedicated moisturising creams during the study.

Patient demographics, baseline clinical data and numbers of patients using local steroid treatment data (week 0) of the 58 patients completing the open-label extension period are displayed in table 1. Patients included had baseline PASI scores between 3.4 and 9.9 at week 0 .

Table 1. Patient demographics, baseline data and use of topical steroids.

\begin{tabular}{|c|c|c|c|c|c|}
\hline Parameter [unit] & Category & Measure & HRO & Placebo & Total \\
\hline \multirow[t]{3}{*}{ Number of subjects } & Total & Number & 28 & 30 & 58 \\
\hline & Male & Number (percent) & $16(57.1)$ & $18(60.0)$ & $34(58.6)$ \\
\hline & Female & Number (percent) & $12(42.9)$ & $12(40.0)$ & $24(41.4)$ \\
\hline Age at informed consent [years] & & Mean (SD) & $46.8(13.6)$ & $51.6(13.7)$ & $49.3(13.8)$ \\
\hline Height $[\mathrm{cm}]$ & & Mean (SD) & $175.5(8.3)$ & $175.0(12.5)$ & $175.2(10.6)$ \\
\hline Body weight $[\mathrm{kg}]$ & & Mean (SD) & $91.8(17.6)$ & $89.0(21.3)$ & $90.4(19.5)$ \\
\hline BMI $\left[\mathrm{kg} / \mathrm{m}^{2}\right]$ & & Mean (SD) & $29.93(5.98)$ & $28.67(3.92)$ & $29.27(5.02)$ \\
\hline PSGA, most frequent score & & Score (percent) & $2(82.1)$ & $2(96.7)$ & $2(89.7)$ \\
\hline BSA & & Mean (SD) & $7.4(4.8)$ & $5.5(2.6)$ & $6.4(3.9)$ \\
\hline DLQI & & Mean (SD) & $9.1(6.3)$ & $8.6(5.3)$ & $8.8(5.7)$ \\
\hline VAS, pruritus & & Mean (SD) & $44(23)$ & $44(24)$ & $44(23)$ \\
\hline VAS, skin pain & & Mean (SD) & $20(20)$ & $19(22)$ & $19(21)$ \\
\hline VAS, singeing & & Mean (SD) & $26(22)$ & $30(24)$ & $28(23)$ \\
\hline VAS, skin disease activity & & Mean (SD) & $48(21)$ & $48(21)$ & $48(21)$ \\
\hline Subjects using local steroids during study & & Number (percent) & $17(61)$ & $15(50)$ & $32(55)$ \\
\hline
\end{tabular}

BMI=Body mass index, PSGA=Physician's Static Global Assessment, BSA=Body Surface Area, VAS=Visual Analogue Scale

Written consent was given by eligible patients who were block randomized using randomly selected block sizes, stratified according to gender, and assigned to either HRO or to the control group (Coconut oil). In both groups, patients received 10 capsules daily, five in the morning and five in the evening in conjunction with a meal. Remaining capsules were counted at clinic visits. At the 26 week visit, patients in both treatment groups were invited to continue on the active treatment regimen.

\subsection{Capsules}

Each HRO capsule contained $292 \mathrm{mg}$ poly-unsaturated fatty acids (total n-3): 22\% eicosapentaenoic acid (EPA, 20:5 n-3) and 66\% docosahexaenoic acid (DHA, 22:6 n-3), where approximately $35 \%$ of both were bound to phospholipids, including phosphatidylcholine. The total daily dose of EPA and DHA was $2.6 \mathrm{~g}$ and the total lipid dose was $5.9 \mathrm{~g}$. Each placebo capsule contained medium chain triglycerides: Coconut oil high in caprylic acid (C8:0) and capric acid (C10:0). The same type $590 \mathrm{mg}$ softgel capsule was filled with active and placebo.

\subsection{Clinical Examinations}

Clinical examinations were performed at screening, baseline and at study weeks $6,12,18,26,46$ and 65 (15 months). Standardised scoring tools in psoriasis were used to 
assess the severity of psoriasis i.e. PASI [7, 8].

\subsection{Patient Reported Data}

DLQI [10] and scores were used to measure: itching (pruritus), pain (skin/joints), singeing and general health conditions (general/skin).

\subsection{Dietary Requirements}

Patients were instructed to discontinue any supplements of cod liver oil, omega-3 and choline for 4 weeks prior to study commencement. Vitamin D, omega-3 and choline supplements were prohibited during the study. Patients were asked not to change their diet including keeping their usual intake of fish in the diet, and to refrain from excessive alcohol intake.

\subsection{Laboratory Analyses}

All serum, plasma and full-blood samples were aliquoted and kept in a locked $-80^{\circ} \mathrm{C}$ freezer until all analyses were performed. Analyses were performed using the same controls and procedures as those routinely used at the hospital laboratory. C-reactive protein (CRP) and safety parameters (liver, kidney, bone marrow) were measured.

\subsection{Statistical Analysis}

Descriptive statistics and frequency counts with percentages were used for continuous endpoint and categorical data, respectively, in order to display the distributions for each endpoint. Only data from patients with complete data until week 65 are included.

\section{Results}

\subsection{Patient Attendance}

Fifty-eight of 64 patients included at week 0 continued through the extension period for a total study period of 65 weeks (15 months). Most patients in both groups chose to participate in the open-label extension period, where 28 of 32 patients who had received HRO in the randomized period continued with HRO, and 30 of 32 who had received placebo were switched to active treatment with HRO (Figure 1). All patients included in the open-label extension completed this period.

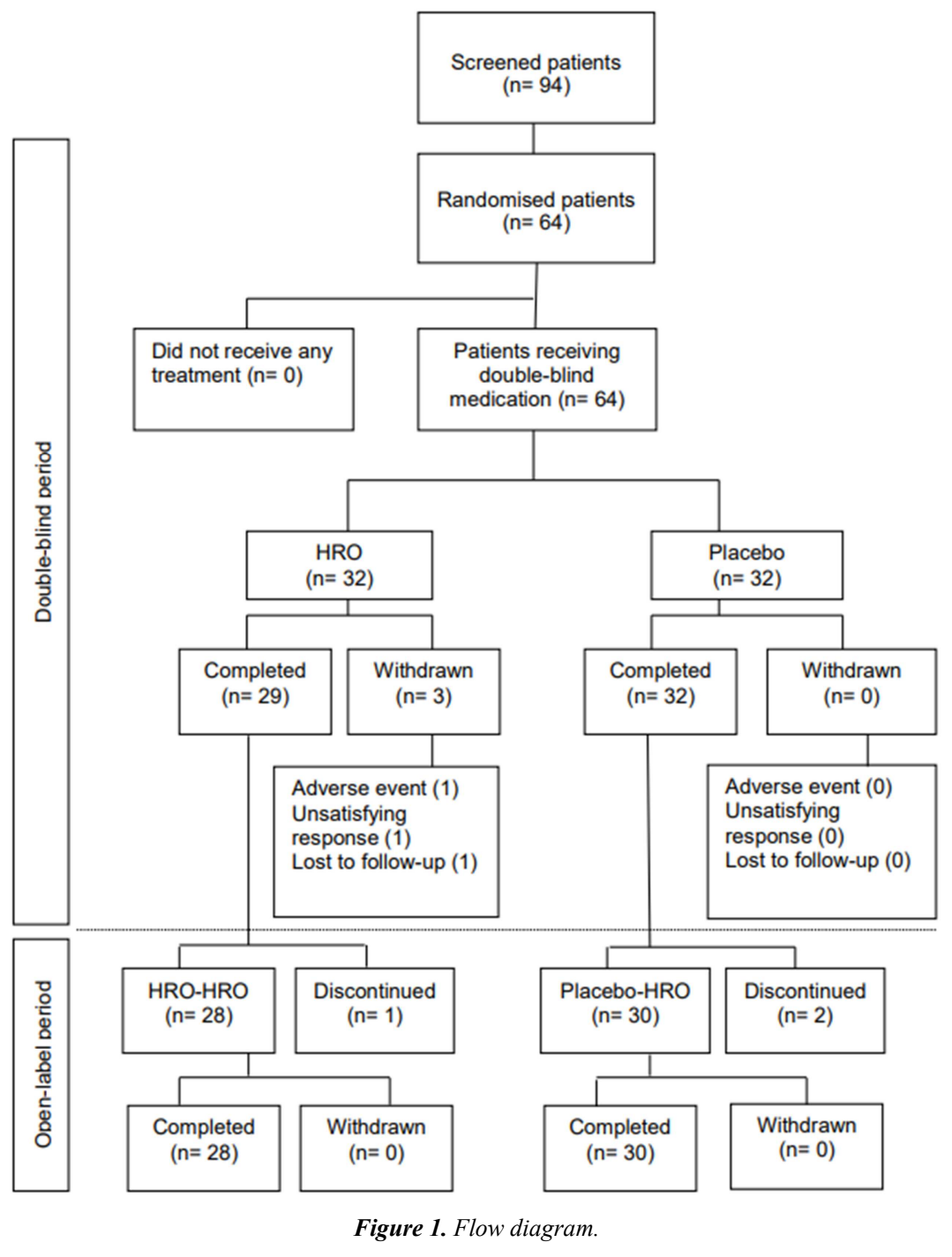




\subsection{Efficacy}

The changes in mean PASI scores $( \pm \mathrm{SD})$ from baseline in the double-blind period was $-2.13 \pm 2.57(35 \%)$ in the HROHRO group $(\mathrm{n}=28)$ and $-0.63 \pm 1.87(11 \%)$ in the placeboHRO group $(n=30)$ and the difference between the groups was statistically significant $(\mathrm{p}=0.0451)$. Over the entire 65 week period (15 months), the PASI reduction was $-3.44 \pm 1.83$ $(56 \%)$ in the HRO-HRO group, $-3.06 \pm 1.69$ (52\%) in the placebo-HRO group (Figure 2) and $53 \%$ in the total population $(\mathrm{n}=58)$.

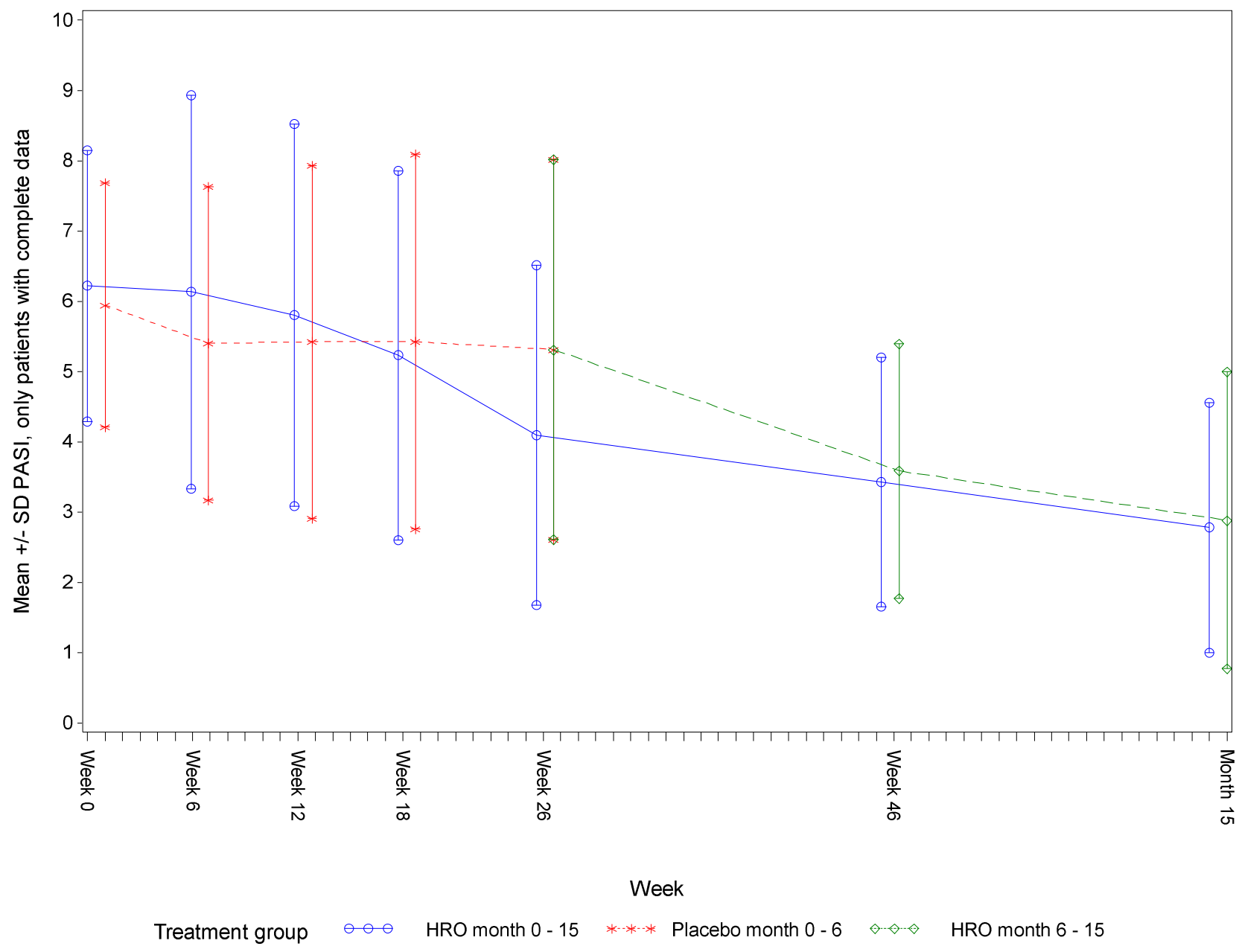

Figure 2. Comparison of mean PASI scores in the HRO-HRO (n=28) and the placebo-HRO $(n=30)$ groups at baseline, weeks 6, 12, 18, 26 (primary endpoint in the randomised control trial), 46 and 65 (15 months). Means and standard deviations are shown. The HRO-HRO group is indicated by a blue line. The placebo-HRO group is indicated by a red dashed line in the randomised period and a green dashed line in the open-label extension period.

The proportions of patients achieving various levels of relative PASI changes from baseline to each visit through the complete course of the study are shown in Figure 3.

The proportion of patients $(n=58)$ achieving a PSGA score of 0 or 1 (clear or almost clear) reached $39.7 \%$ at 65 weeks. Figure 4 illustrates the development of patient proportions with different PSGA scores during the study. All patients had PSGA scores $\geq 2$ and $\leq 4$ at inclusion, and after 65 weeks no patient had a PSGA score higher than 3 . In total, $46.6 \%$ of patients had a reduction in their PSGA score.

The mean BSA scores $( \pm \mathrm{SD})$ and the changes in scores $( \pm \mathrm{SD})$ from baseline at each visit for all participants are displayed in table 2. The change in BSA score from baseline in the double-blind period was $-1.79 \pm 4.80$ (mean \pm standard deviation; SD) in the HRO-HRO group $(n=28)$ and $-0.02 \pm 3.18$ in the placebo-HRO group $(n=30)$.
Over the entire 65-week period (15 months), the change in BSA was $-3.38 \pm 4.63$ in the HRO-HRO group and $2.23 \pm 2.98$ in the placebo-HRO group i.e. the mean values were reduced by $46 \%$ and $41 \%$, respectively, and $44 \%$ in the total population $(\mathrm{n}=58)$.

Table 2. Mean BSA scores $( \pm S D)$ and mean change from baseline $( \pm S D)$ in patients completing the 15 month visit.

\begin{tabular}{llllll}
\hline \multirow{2}{*}{ Visit } & \multicolumn{2}{c}{ BSA } & \multicolumn{3}{c}{ Change from baseline } \\
\cline { 2 - 6 } & N & Mean & SD & Mean & SD \\
\hline Week 0 & 58 & 6.41 & 3.89 &. &. \\
Week 6 & 58 & 6.71 & 4.32 & 0.30 & 2.55 \\
Week 12 & 58 & 6.65 & 4.79 & 0.24 & 3.79 \\
Week 18 & 58 & 6.39 & 4.76 & -0.01 & 3.86 \\
Week 26 & 58 & 5.53 & 4.36 & -0.87 & 4.11 \\
Week 46 & 58 & 4.70 & 3.81 & -1.71 & 3.06 \\
Month 15 & 58 & 3.62 & 3.46 & -2.79 & 3.87 \\
\hline
\end{tabular}




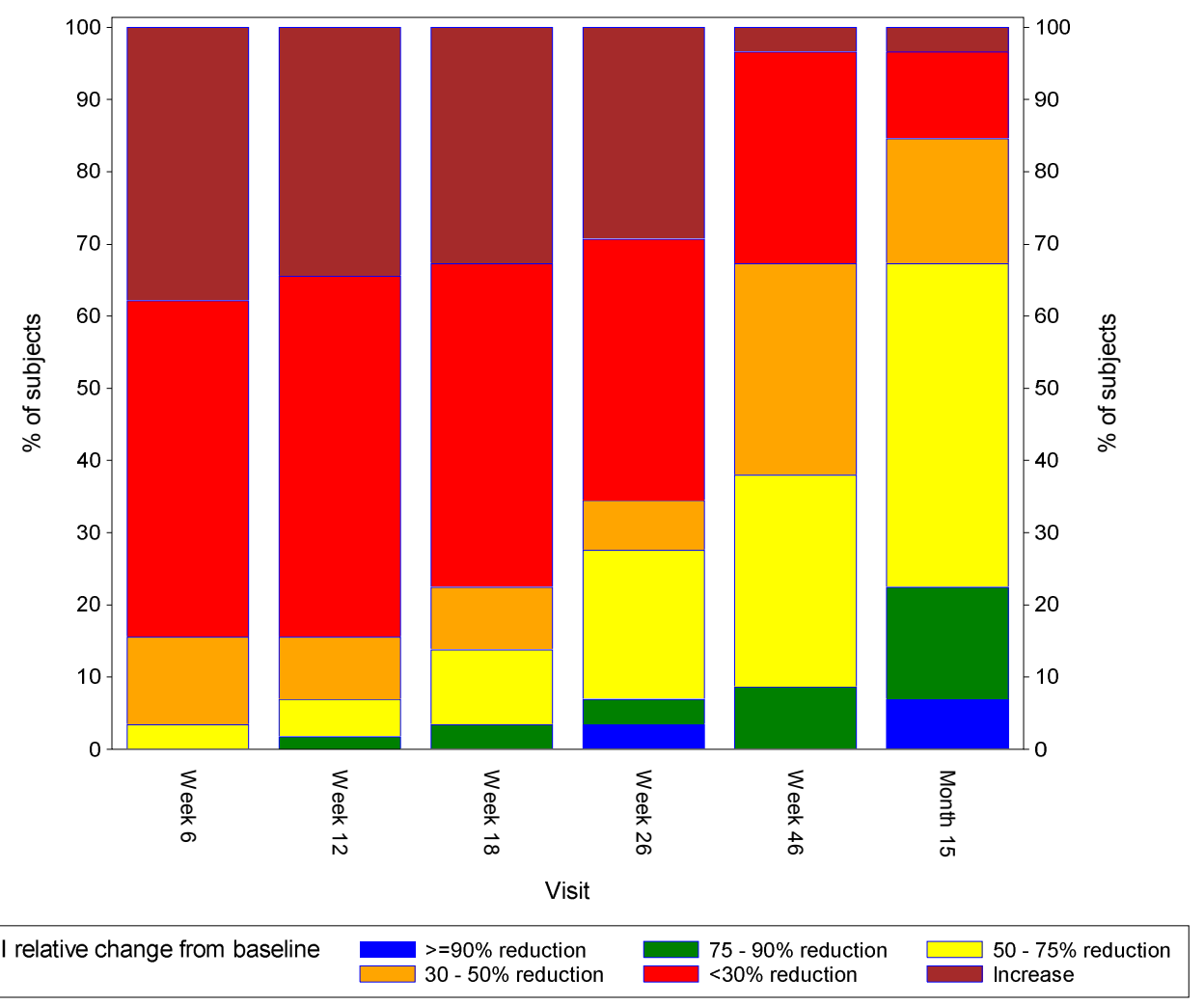

Figure 3. Proportions of patients with relative changes of $\geq 90 \%$ reduction in PASI (blue), 75-90\% reduction (green), 50-75\% reduction (yellow), 30-50\% reduction (orange), $<30 \%$ reduction (red) and increases (dark red) at clinic visits weeks 6, 12, 18, 26, 46 and 65. Percentages are calculated using the total population completing the open-label extension period $(n=58)$.

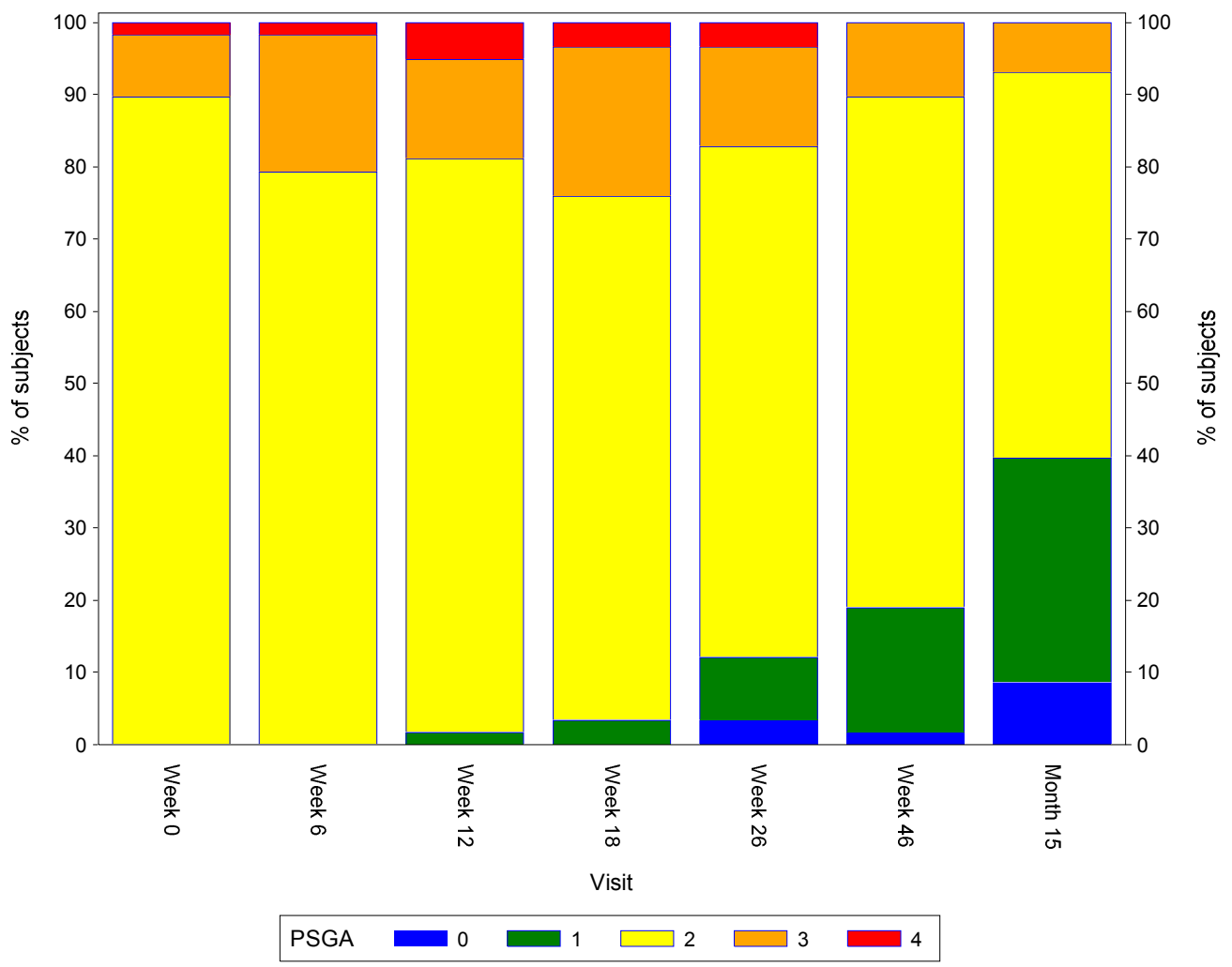

Figure 4. Proportions of patients with different PSGA scores: 0 (blue), 1 (green), 2 (yellow), 3 (orange) and 4 (red) at clinic visits, weeks 0, 6, 12, 18, 26, 46 and 65. Percentages are calculated using the total population completing the open-label extension period $(n=58)$. 
Reductions in patient reported VAS scores of skin disease activity, pruritus, skin pain and singeing were observed in the open-label extension population $(\mathrm{n}=58)$. The mean VAS scores $( \pm \mathrm{SD})$ and the changes in scores $( \pm \mathrm{SD})$ from baseline at each visit for all participants are displayed in table 3. Over the entire 65-week period (15 months), the changes in VAS scores in the HRO-HRO group were: skin disease activity $-21 \pm 21$ (43\%), pruritus $-23 \pm 24(51 \%)$, skin pain $-12 \pm 17(61 \%)$ and singeing $15 \pm 20(57 \%)$, whereas in the placebo-HRO group the changes were: skin disease activity $-20 \pm 23(42 \%)$, pruritus $-19 \pm 27$ (43\%), skin pain $-7 \pm 17(37 \%)$ and singeing $-15 \pm 20$ (50\%). In the total population, the percent reductions in mean scores from baseline to study-end were: skin disease activity $42 \%$, pruritus $46 \%$, skin pain $49 \%$ and singeing $52 \%$.

Table 3. Mean VAS scores and standard deviations (SD) for skin disease activity, pruritus, skin pain and singeing, and the changes in scores ( \pm SD) from baseline at each visit in patients completing the 15 month visit $(n=58)$.

\begin{tabular}{|c|c|c|c|c|c|c|c|c|c|}
\hline \multirow{3}{*}{ Visit } & \multirow{3}{*}{$\mathbf{N}$} & \multicolumn{4}{|c|}{ Skin disease activity } & \multicolumn{4}{|c|}{ Pruritus } \\
\hline & & \multicolumn{2}{|c|}{ Score } & \multicolumn{2}{|c|}{ Change from baseline } & \multicolumn{2}{|c|}{ Score } & \multicolumn{2}{|c|}{ Change from baseline } \\
\hline & & Mean & SD & Mean & SD & Mean & SD & Mean & SD \\
\hline Week 0 & 58 & 48 & 21 & . & . & 44 & 23 & . & . \\
\hline Week 6 & 58 & 41 & 23 & -7 & 19 & 37 & 25 & -7 & 20 \\
\hline Week 12 & 58 & 42 & 24 & -6 & 23 & 38 & 25 & -6 & 23 \\
\hline Week 18 & 58 & 43 & 26 & -5 & 20 & 38 & 26 & -7 & 23 \\
\hline Week 46 & 58 & 28 & 23 & -20 & 21 & 24 & 23 & -20 & 26 \\
\hline Month 15 & 58 & 28 & 23 & -20 & 22 & 24 & 23 & -20 & 26 \\
\hline
\end{tabular}

Table 3. Continued.

\begin{tabular}{|c|c|c|c|c|c|c|c|c|c|}
\hline \multirow{3}{*}{ Visit } & \multirow{3}{*}{$\mathbf{N}$} & \multicolumn{4}{|c|}{ Skin pain } & \multicolumn{4}{|c|}{ Singeing } \\
\hline & & \multicolumn{2}{|c|}{ Score } & \multicolumn{2}{|c|}{ Change from baseline } & \multicolumn{2}{|c|}{ Score } & \multicolumn{2}{|c|}{ Change from baseline } \\
\hline & & Mean & SD & Mean & SD & Mean & SD & Mean & SD \\
\hline Week 0 & 58 & 19 & 21 & . & . & 28 & 23 & . & . \\
\hline Week 6 & 58 & 15 & 21 & -4 & 17 & 21 & 23 & -7 & 18 \\
\hline Week 12 & 58 & 20 & 24 & 0 & 20 & 27 & 29 & -1 & 24 \\
\hline Week 18 & 58 & 20 & 23 & 1 & 20 & 27 & 25 & -1 & 21 \\
\hline Week 46 & 58 & 11 & 17 & -9 & 20 & 15 & 20 & -13 & 19 \\
\hline Month 15 & 58 & 10 & 15 & -9 & 17 & 14 & 18 & -15 & 20 \\
\hline
\end{tabular}

The mean DLQI scores $( \pm \mathrm{SD})$ and the changes in scores $( \pm \mathrm{SD})$ from baseline at each visit for all participants are displayed in table 4 . The change in DLQI score from baseline in the double-blind period was $-2.6 \pm 6.0$ (mean \pm standard deviation; SD) in the HRO-HRO group $(\mathrm{n}=28)$ and $-0.8 \pm 4.3$ in the placebo-HRO group $(n=30)$. Over the entire 65-week period (15 months), the changes in DLQI scores were $4.9 \pm 5.0$ in the HRO-HRO group and $-4.1 \pm 5.4$ in the placeboHRO group. In the open-label extension population $(n=58)$, the reductions in mean values from baseline to study-end were $54 \%$ in the HRO-HRO group and $47 \%$ in the placeboHRO group. In the total population the reduction was $51 \%$.

Table 4. Mean DLQI scores $( \pm S D)$ and mean change from baseline $( \pm S D)$ in patients completing the 15 month visit.

\begin{tabular}{llllll}
\hline \multirow{2}{*}{ Visit } & DLQI & \multicolumn{3}{c}{ Change from baseline } \\
\cline { 2 - 6 } & N & Mean & SD & Mean & SD \\
\hline Week 0 & 58 & 8.8 & 5.7 & $\cdot$ &. \\
Week 6 & 58 & 7.2 & 5.6 & -1.6 & 3.8 \\
Week 12 & 58 & 7.7 & 5.8 & -1.1 & 4.4 \\
Week 18 & 58 & 8.0 & 5.8 & -0.8 & 4.9 \\
Week 26 & 58 & 7.2 & 5.6 & -1.7 & 5.2 \\
Week 46 & 58 & 5.1 & 4.4 & -3.7 & 4.9 \\
Month 15 & 58 & 4.3 & 4.2 & -4.5 & 5.2 \\
\hline
\end{tabular}

\subsection{Safety}

Adverse events were recorded throughout the study and most patients experienced one or more adverse event in the 15 month study duration. During the open-label extension, 25 (43\%) patients experienced adverse events, 11 (39\%) in the HRO-HRO group and 14 (47\%) in the placebo-HRO group. One serious adverse event was reported at the 65-week visit when a patient experienced atrial fibrillation which was not considered related to treatment.

\section{Discussion}

The longer-term efficacy and safety of HRO supplementation in patients with plaque psoriasis was explored in this 39-week active-treatment extension period in terms of clinical variables i.e. PASI, PSGA, BSA, DLQI, VAS scores (pruritus, skin pain, singeing and general skin condition) and adverse events.

In the preceding randomised period (26 weeks), a statistically significant mean reduction in PASI in the HROgroup versus the placebo group was observed [6]. The clinical relevance of the observed mean PASI improvement $[11,12]$ during the randomised period could not be readily 
concluded. However, during the extension period, all patients received active treatment $(n=58)$, and the mean PASI scores continued to decrease with an improvement in mean PASI of $53 \%$ from baseline to week 65 . The proportion of patients with at least a 50\% reduction in PASI at week 65 compared to baseline was $67.2 \%$. Secondary efficacy variables showed the same decrease: PSGA at 0 or 1 from $0 \%$ to $39.7 \%$ of patients; a reduction of mean BSA value of $44 \%$; reductions in mean VAS scores of $42 \%$ for skin disease activity, $46 \%$ for pruritus, $49 \%$ for skin pain and $52 \%$ for singeing. The patients treated with $\mathrm{HRO}$ for the entire 15 months experienced an even greater reduction in these secondary endponts; reductions in mean VAS scores of $43 \%$ for skin disease activity, $51 \%$ for pruritus, $61 \%$ for skin pain and $57 \%$ for singeing. Even more important were the findings showing a reduction in mean values from baseline to study-end in DLQI of 51\% (HRO-HRO group: $54 \%$ and placebo-HRO group: $47 \%$ ). In absolute terms the reductions in mean DLQI from baseline to the end of the study (15 month) were 4.5 points in the total population, 4.9 points in the HRO-HRO group and 4.1 points in the placebo-HRO group. Corresponding numbers from baseline to the end of the randomized part of the study ( 26 weeks) showed a reduction of only $1.7,2.6$ and 0.8 points for all patients, HRO-HRO patients and placebo-HRO patients, respectively. This has some important implications. An absolute decrease of 4 points in the DLQI-score has been proposed as the minimal clinically important difference (MCID) for most types of skin diseases [13-15]. Thus, an improvement of this magnitude will be considered as a relevant improvement by most patients. These reductions in absolute DLQI scores are encouraging since the mean baseline DLQI score was only 8.8 for all participants $(\mathrm{n}=58), 9.1$ in the HRO-HRO group $(n=28)$ and 8.6 in the placebo-HRO group $(n=30)$, but were nonetheless high enough to reflect a clinically relevant improvement even at the lower end of the scale.

Adverse events were recorded throughout the study and most patients experienced one or more adverse event during the 65-week study period. However, no serious adverse reactions occurred during the full course of the study from randomisation to week 65 . Additionally, more than $90 \%$ of the randomised patients ( 58 out of 64 ) completed the 65 week visit. This is considered a high completion ratio for a study this long and indicates that the treatment was well tolerated.

\section{Conclusions}

The lack of a control group during the active-treatment extension, and hence the lack of a statistical test, represents a limitation. However, the similarity of disease improvements as measured by the investigator (PASI, PSGA and BSA) and patient reported outcome measures (VAS and DLQI), as well as their magnitude, strengthens the assumption that HRO improves psoriasis when administered for a long time. Thus, HRO may be a promising new, oral treatment option for nonsevere psoriasis, where there have been few treatment advances in recent years as most of the new drugs are indicated for the treatment of moderate to severe psoriasis [16-18]. The results of this study should be confirmed in a large multicentre study.

\section{Declarations}

\section{Funding}

This study was sponsored by Arctic Nutrition AS.

\section{Conflict of Interest}

The authors declare that they have no competing interests.

\section{Ethics Approval}

This study was performed in line with the principles of the Declaration of Helsinki. Approval was granted by the Regional Committees for Medical and Health Research Ethics in western Norway (REK Vest, date: 21.06.2020/No. 2017/938).

\section{Consent to Participate}

Informed consent was obtained from all individual participants included in the study.

\section{Consent for Publication}

Patients signed informed consent regarding publishing their data.

\section{Authors' Contributions}

All authors read and approved the final manuscript and are responsible for correctness of the statements provided in the manuscript.

\section{Acknowledgements}

The study was conducted with support from Forskningsenhet for helseundersøkelser (FHU) under the supervision of professor Fransisco Real. FHU was established with financial contributions from Trond Mohn Stiftelse (TMS).

Support in blood testing from Laboriatorium at the Dermatovenerology department Haukeland University Hospital from Senior Biomedical Laboratory Scientist Bente Torvund.

\section{References}

[1] Boehncke W-H. Systemic Inflammation and Cardiovascular Comorbidity in Psoriasis Patients: Causes and Consequences. Front Immunol. 2018; 9: 579.

[2] Bjørndal B, Strand E, Gjerde J, Bohov P, Svardal A, Diehl $\mathrm{BW}$, et al. Phospholipids from herring roe improve plasma lipids and glucose tolerance in healthy, young adults. Lipids Health Dis. 2014 May 17; 13: 82. 
[3] Cook CM, Hallaråker H, Sæbø PC, Innis SM, Kelley KM, Sanoshy KD, et al. Bioavailability of long chain omega-3 polyunsaturated fatty acids from phospholipid-rich herring roe oil in men and women with mildly elevated triacylglycerols. Prostaglandins Leukot Essent Fatty Acids. 2016; 111: 17-24.

[4] Khorsan R, Crawford C, Ives JA, Walter AR, Jonas WB. The effect of omega-3 fatty acids on biomarkers of inflammation: a rapid evidence assessment of the literature. Mil Med. 2014 Nov; 179 (11 Suppl): 2-60.

[5] Bannenberg G, Serhan CN. Specialized pro-resolving lipid mediators in the inflammatory response: An update. Biochim Biophys Acta. 2010 Dec; 1801 (12): 1260-73.

[6] Tveit KS, Brokstad KA, Berge RK, Sæbø PC, Hallaråker H, Brekke S, et al. A Randomized, Double-blind, Placebocontrolled Clinical Study to Investigate the efficacy of Herring Roe Oil for treatment of Psoriasis. Acta Derm Venereol. 2020 May 28; 100 (10): adv00154.

[7] Fredriksson T, Pettersson U. Severe psoriasis--oral therapy with a new retinoid. Dermatologica. 1978; 157 (4): 238-44.

[8] Feldman SR, Krueger GG. Psoriasis assessment tools in clinical trials. Ann Rheum Dis. 2005 Mar; 64 Suppl 2: ii65-68; discussion ii69-73.

[9] Armstrong AW, Robertson AD, Wu J, Schupp C, Lebwohl MG. Undertreatment, treatment trends, and treatment dissatisfaction among patients with psoriasis and psoriatic arthritis in the United States: findings from the National Psoriasis Foundation surveys, 2003-2011. JAMA Dermatol. 2013 Oct; 149 (10): 1180-5.

[10] Finlay AY, Khan GK. Dermatology Life Quality Index (DLQI)--a simple practical measure for routine clinical use. Clin Exp Dermatol. 1994 May; 19 (3): 210-6.
[11] Mrowietz U, Kragballe K, Reich K, Spuls P, Griffiths CEM, Nast A, et al. Definition of treatment goals for moderate to severe psoriasis: a European consensus. Arch Dermatol Res. 2011 Jan; 303 (1): 1-10.

[12] Puzenat E, Bronsard V, Prey S, Gourraud P-A, Aractingi S, Bagot $\mathrm{M}$, et al. What are the best outcome measures for assessing plaque psoriasis severity? A systematic review of the literature. J Eur Acad Dermatol Venereol JEADV. 2010 Apr; 24 Suppl 2: 10-6.

[13] Basra MKA, Salek MS, Camilleri L, Sturkey R, Finlay AY. Determining the Minimal Clinically Important Difference and Responsiveness of the Dermatology Life Quality Index (DLQI): Further Data. Dermatology. 2015; 230 (1): 27-33.

[14] Basra MKA, Fenech R, Gatt RM, Salek MS, Finlay AY. The Dermatology Life Quality Index 1994-2007: a comprehensive review of validation data and clinical results. Br J Dermatol. 2008 Nov; 159 (5): 997-1035.

[15] Khilji FA, Gonzalez M, Finlay AY. Clinical meaning of change in Dermatology Life Quality Index scores. Br J Dermatol. 2002; 147 (50).

[16] Armstrong AW, Read C. Pathophysiology, Clinical Presentation, and Treatment of Psoriasis: A Review. JAMA. 2020 May 19; 323 (19): 1945-60.

[17] Greb JE, Goldminz AM, Elder JT, Lebwohl MG, Gladman DD, Wu JJ, et al. Psoriasis. Nat Rev Dis Primer. 2016 24; 2: 16082 .

[18] Sbidian E, Chaimani A, Afach S, Doney L, Dressler C, Hua C, et al. Systemic pharmacological treatments for chronic plaque psoriasis: a network meta-analysis. Cochrane Database Syst Rev. 2020 09; 1: CD011535. 